\title{
Phase transitions in the three-dimensional $Z(N)$ models
}

\section{Oleg Borisenko}

BITP, National Academy of Science of Ukraine, 003680 Kiev, Ukraine

E-mail: olegdbitp.kiev.ua

\section{Vladimir Chelnokov*}

BITP, National Academy of Science of Ukraine, 003680 Kiev, Ukraine

E-mail: vchelnokovdi.ua

\section{Gennaro Cortese}

Instituto de Física Teórica UAM/CSIC, Cantoblanco, E-28049 Madrid, Spain,

\& Departamento de Física Teórica, Universidad de Zaragoza, E-50009 Zaragoza, Spain

E-mail: cortesedunizares

\section{Mario Gravina}

Dipartimento di Fisica, Università della Calabria,

\& INFN - Gruppo Collegato di Cosenza, I-87036 Rende, Italy

E-mail: gravinadcs.infn.it

\section{Alessandro Papa}

Dipartimento di Fisica, Università della Calabria,

\& INFN - Gruppo Collegato di Cosenza, I-87036 Rende, Italy

E-mail: papaecs.infn.it

\section{Ivan Surzhikov}

BITP, National Academy of Science of Ukraine, 003680 Kiev, Ukraine

E-mail: i_van_godinbox.ru

\begin{abstract}
Phase transitions in zero-temperature $3 D Z(N)$ lattice gauge theories are studied. We use a cluster algorithm defined for the dual formulation of the models. We also attempt to explain the nature of the intermediate continuously symmetric phase, which appears for $N>5$. The critical indices are calculated. The results obtained are used to study the scaling of critical points with $N$, as well as the scaling of finite-temperature critical points with the lattice size in the time direction, $N_{T}$.
\end{abstract}

31st International Symposium on Lattice Field Theory LATTICE 2013

July 29 - August 3, 2013

Mainz, Germany

\footnotetext{
* Speaker.
} 


\section{Introduction}

The properties of the phase transitions in $Z(N)$ models are interesting both by themselves and due to their connection with the deconfining transitions in $S U(N)$ gauge theories, $Z(N)$ being the center subgroup of $S U(N)$. In this paper we study the $Z(N)$ lattice gauge theory (LGT) at zero temperature. In the most general form, the action of the isotropic $Z(N)$ LGT can be written as

$$
S_{\text {gauge }}=\frac{1}{2} \sum_{x} \sum_{n<m} \sum_{k=1}^{N-1} \beta_{k} \exp \left(\frac{2 \pi i k}{N}\left(s_{n}(x)+s_{m}\left(x+e_{n}\right)-s_{n}\left(x+e_{m}\right)-s_{m}(x)\right)\right)
$$

where $e_{n}, n=1,2,3$, denotes the unit vector in the $n$-th direction. Similarly, the most general action of the $Z(N)$ spin model is given by

$$
S_{\text {spin }}=\frac{1}{2} \sum_{x} \sum_{n} \sum_{k=1}^{N-1} \beta_{k} \exp \left(\frac{2 \pi i k}{N}\left(s(x)-s\left(x+e_{n}\right)\right)\right) .
$$

In both cases we used the convention

$$
\beta_{k}=\beta_{-k}=\beta_{k+N}>0 .
$$

The standard Potts model corresponds to the choice when all $\beta_{k}$ are equal. Then, the sum over $k$ reduces to a delta-function on the $Z(N)$ group. The conventional vector model corresponds to $\beta_{k}=0$ for all $k \neq 1, N-1$. For $N=2,3$ the Potts and vector models are equivalent.

Two-dimensional (2D) standard and vector $Z(N)$ LGTs are exactly solvable both in the finite volume and in the thermodynamic limit. They exhibit no phase transition at any finite value of the coupling constant $\beta$. For $3 D Z(N)$ models no exact solution is known. While the phase structure of the general model defined by $(\mathbb{L} \cdot \mathbb{C})$ remains unknown, it is well established that Potts and vector models with only $\beta_{1}$ non-vanishing have one phase transition from a confining phase to a phase with vanishing string tension [U, [1, []].

The duality relation between $3 D Z(N)$ gauge and spin models allows to establish the order of phase transition in $3 D Z(N)$ LGT using the results obtained for spin models. Since $Z(2)$ LGT is equivalent to the Ising model, its critical behavior is well known (see Refs. [四] and references therein). Generally, the $Z(N)$ global symmetry of the finite-temperature $4 D S U(N)$ gauge theory motivated thorough investigations, both analytical and numerical, of the $3 D$ spin models, especially for $N=2,3$ [ [G, 目] (for more recent studies, see [ $[$ ] and references therein). The $3 D$ Potts models for $N>3$ have been simulated in [[]] and studied by means of the high-temperature expansion in [9].

Surprisingly, much less is known about the critical behavior of $Z(N)$ vector LGTs when $N>4$. They have been studied numerically in [ए]] up to $N=20$ on symmetric lattices with size $L \in[4-16]$. It was confirmed that zero-temperature models possess a single phase transition which disappears in the limit $N \rightarrow \infty$. A scaling formula proposed in [ए0] shows that the critical coupling diverges like $N^{2}$ for large $N$. Thus, the $U(1)$ LGT has a single confined phase in agreement with theoretical results [U]]. We are not aware, however, of any detailed study of the critical behavior of the vector models with $N \geq 4$ in the vicinity of this single phase transition. Slightly more is known about the critical properties of $Z(N)$ vector spin models. In particular, it has been suggested that all vector spin models exhibit a single second order phase transition [10]]. 
An especially detailed study was performed on the $Z(6)$ model, because the $Z(6)$ global symmetry appears as an effective symmetry of the $Z(3)$ antiferromagnetic Potts model [[13, [4]]. The computed critical indices suggest that the $Z(6)$ vector model belongs to the universality class of the $3 D$ $X Y$ model. An interesting feature of the $Z(6)$ model and, possibly, of all vector models with $N>4$, is the appearance of an intermediate rotationally symmetric region below the critical temperature of the second order phase transition. The mass gap, however, was found to be rather small, but non-vanishing in this region [[3]]. Combined with a renormalization group (RG) study, the analysis concluded that this intermediate region presents a crossover to a low-temperature massive phase, where the discreteness of $Z(6)$ plays an essential role [144].

The main goal of the present work is to fill the gap in our knowledge about the critical behavior of the $3 D Z(N)$ LGTs. Another motivation comes from our recent studies of the deconfinement

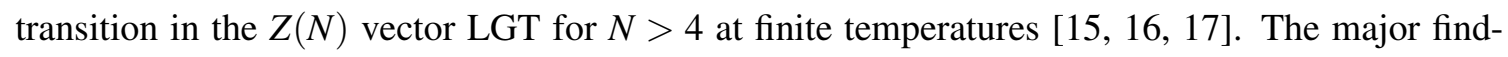
ings of these papers was the demonstration of two phase transitions of the Berezinskii-KosterlitzThouless type and the existence of an intermediate massless phase. The critical indices at these transitions have been found to coincide with the indices of the $2 D$ vector spin models. An interesting question then arises regarding the construction of the continuum limit of the finite-temperature models in the vicinity of the critical points. For this to accomplish it might be useful, and even necessary, to know the scaling of quantities such as string tension, correlation length, etc. near the critical points of the corresponding zero temperature models.

\section{Numerical results}

To study the phase transitions in $3 D Z(N)$ LGTs, it turns out to be more convenient to simulate the dual spin model, whose action is given in $(\mathbb{L 2} 2)$. The equations for obtaining the coupling constants for the dual spin model are given in [ए]]. Simulations were performed by means of a cluster algorithm on symmetric lattices $L^{3}$ with periodic $\mathrm{BC}$ and $L$ in the range $8-96$. For each Monte Carlo run the typical number of generated configurations was $2.5 \cdot 10^{6}$, the first $10^{5}$ of them being discarded to ensure thermalization. Measurements were taken after every 10 whole lattice updates and error bars were estimated by the jackknife method combined with binning. The following observables were used:

- complex magnetization $M_{L}=\left|M_{L}\right| e^{i \psi}, M_{L}=\sum_{x \in \Lambda} \exp \left(\frac{2 \pi i}{N} s(x)\right)$;

- population $S_{L}, S_{L}=\frac{N}{N-1}\left(\frac{\max _{i=0, N-1} n_{i}}{L^{3}}-\frac{1}{N}\right)$, where $n_{i}$ is number of $s(x)$ equal to $i$;

- susceptibilities of $M_{L}$ and $S_{L}: \chi_{L}^{(M)}, \chi_{L}^{(S)}, \chi_{L}^{(\cdot)}=L^{2}\left(\left\langle\cdot{ }^{2}\right\rangle-\langle\cdot\rangle^{2}\right)$;

- Binder cumulant $U_{L}^{(M)}=1-\frac{\left\langle\left|M_{L}\right|^{4}\right\rangle}{3\left\langle\left|M_{L}\right|^{2}\right\rangle^{2}}$.

We computed also the heat capacity in the vicinity of the critical points.

To obtain the critical couplings, the Binder cumulant crossing method described in [20] was used. In particular, we computed by Monte Carlo simulations the Binder cumulant $U_{L}^{(M)}$ and its first three derivatives with respect to $\beta$ for the different lattice sizes, thus allowing to build the function $U_{L}^{(M)}(\beta)$ in the region near the transition. Then, we looked for the value of $\beta$ at which the curves 

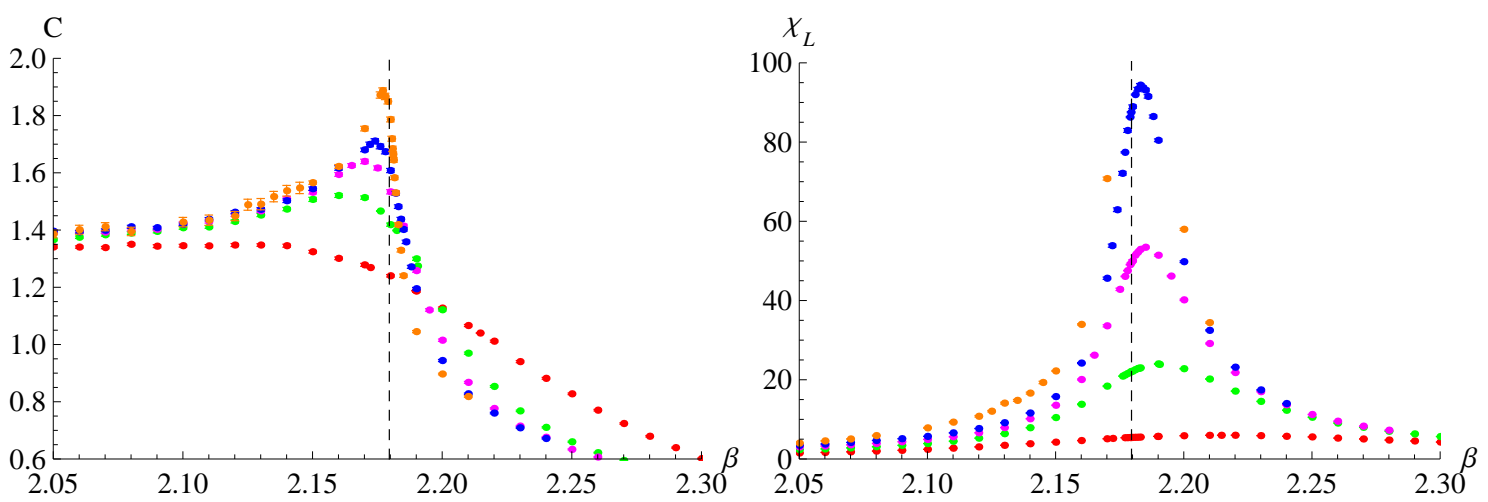

Figure 1: Heat capacity (left) and susceptibility $\chi_{L}^{(M)}$ (right) versus $\beta$ for the $3 D Z(5)$ vector model on lattices with $L=8$ (red), 16 (green), 24 (violet), 32 (blue) and 64 (orange). The vertical dashed line shows the location of the critical point. The error bars are smaller than symbols.

$U_{L}^{(M)}(\beta)$ related to the different lattice sizes $L$ "intersect". In fact, the critical coupling $\beta_{\mathrm{c}}$ was estimated as the value of $\beta$ at which $U_{L}^{(M)}(\beta)$ exhibits the least dispersion over lattice sizes ranging from $L=16$ to $L=96$. To check critical couplings we have compared them with the results of the SDA RG [R] and found out that the values obtained from RG and from Monte Carlo simulations are very close.

The critical coupling values for the $3 D Z(N>4)$ vector models can be fitted with the formula

$$
\beta_{\mathrm{c}}=\frac{A}{1-\cos \left(\frac{2 \pi}{N}\right)}+C\left(1-\cos \left(\frac{2 \pi}{N}\right)\right),
$$

being a generalization of the critical coupling scaling given in [ए]], with following parameters $A=1.50122(7), C=0.0096(5), \chi^{2} /$ d.o.f. $=13.1$. Despite the large $\chi^{2}$, probably due to the underestimation of the error bars of critical couplings, the proposed function nicely interpolates data over a large interval of values of $N$.

The procedure to determine the critical index $v$ is also inspired by Ref. [RO]: for each lattice size $L$ the known function $U_{L}^{(M)}(\beta)$ is used to determine $d U_{L}^{(M)}(\beta) / d \beta$; from this, the derivative of $U_{L}^{(M)}$ with respect to the rescaled coupling $x=\left(\beta-\beta_{\mathrm{c}}\right) L^{1 / v}$ can be calculated,

$$
\frac{d U_{L}^{(M)}}{d x}=\frac{d U_{L}^{(M)}}{d \beta} L^{1 / v}
$$

The best estimate of $v$ is found by minimizing the deviation of $d U_{L}^{(M)} / d x$ with respect to a constant value. The minimization can be done at $\beta_{\mathrm{c}}$ or at any other value $\beta_{\mathrm{f}} \approx \beta_{\mathrm{c}}$ defined as the point where $U_{L}^{(M)}$ on a given lattice becomes equal to some fixed value. The resulting values for $v$, do not differ within error bars.

The critical index $\alpha$, determined from the $v$ values obtained in the previous subsection by means of the relation $\alpha=2-d v$, gets negative values for all $N \geq 5$, meaning that the transition is of order higher than two. In fact, these negative values are very close to that of the $3 D X Y$ model [20]. However, the plots of the heat capacity (see Fig. W(left)) clearly show that it diverges in the vicinity of the critical point. Moreover, the maxima of the heat capacity and of the susceptibility $\chi_{L}^{(M)}$ approach the critical point from different sides (see Figs. W). 
This suggests that a different value for the index $v$ can be found if a finite-size scaling (FSS) analysis is done on the peak values of the heat capacity, using as fitting function

$$
C(L)=A L^{\alpha / v} .
$$

After $\alpha / v$ is extracted, from the relation $\alpha=2-d v$ the value of $v$ can be obtained.

We see that while for $N=2,4$ the resulting $v$ agrees with the value of $v$ in the $3 D$ Ising model, it is not so for $N>4$. The most important fact is, however, that in all cases the $v$ indices obtained this way are close to $v \approx 0.63$ - the critical index for the Ising model. The difference between $v$ indices obtained from the $U_{L}^{(M)}$ cumulants and from the heat capacity leads us to conclude that we have two kinds of singularity depending on whether one approaches the critical coupling from above (3D XY model-like singularity) or from below ( $3 D$ Ising universality class), for $N>4$.

Finally, the critical indices $\beta / v$ and $\gamma / v$ can be extracted from the FSS analysis of the magnetization $M_{L}$ and its susceptibility $\chi_{L}^{(M)}$, according to the following fitting functions,

$$
M_{L}=A_{1} L^{-\beta / v}, \chi_{M_{L}}=A_{2} L^{\gamma / v}\left(1+B_{2} L^{-\delta / v}\right) .
$$

The critical index $\eta$ will then be given by $2-\gamma / v$ and the hyperscaling relation $d=2 \beta / v+\gamma / v$ must be satisfied with $d=3$. The $\delta$ exponent was fixed to be 0.53 - the value for the $X Y$ model. When we take $\delta$ in the range 0.4-0.7 the change of the critical index $\gamma / v$ remains smaller than the error estimates, so our results do not depend much on the exact value of $\delta$. On the other hand, without this second order correction $\eta$ value becomes much smaller (see [एप] for comparison).

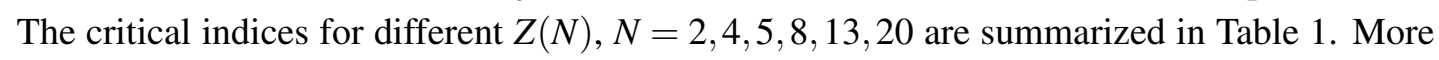
detailed results with comparison of the different methods can be found in [ए9].

Table 1: Critical couplings and critical indices for $3 D Z(N)$ models: ( $v_{\text {Binder }}$ corresponds to the $v$ index obtained from the Binder cumulant derivatives, $v_{\text {heat }}-$ the $v$ index obtained from the heat capacity, $d=$ $2 \beta / v+\gamma / v$ and should be equal to 3 ).

\begin{tabular}{|c|c|c|c|c|c|c|}
\hline$N$ & $\beta_{\mathrm{c}}$ & $v_{\text {Binder }}$ & $v_{\text {heat }}$ & $\beta / v$ & $\gamma / v$ & $\eta$ \\
\hline 2 & $0.761395(4)$ & $0.6306(8)$ & $0.6143(6)$ & $0.504(2)$ & $1.975(8)$ & $0.025(18)$ \\
4 & $1.52276(4)$ & $0.62933(12)$ & $0.6168(14)$ & $0.493(2)$ & $1.951(14)$ & $0.049(14)$ \\
5 & $2.17961(10)$ & $0.6681(8)$ & $0.6360(6)$ & $0.5088(7)$ & $1.971(12)$ & $0.029(12)$ \\
6 & $3.00683(7)$ & $0.6756(16)$ & $0.6360(10)$ & $0.5052(9)$ & $1.970(5)$ & $0.030(5)$ \\
8 & $5.12829(13)$ & $0.6748(2)$ & $0.6336(3)$ & $0.5083(8)$ & $1.966(4)$ & $0.034(4)$ \\
13 & $13.1077(3)$ & $0.6723(17)$ & $0.6340(4)$ & $0.5092(11)$ & $1.971(7)$ & $0.029(7)$ \\
20 & $30.6729(5)$ & $0.6739(7)$ & $0.6314(3)$ & $0.5034(7)$ & $1.99(6)$ & $0.01(6)$ \\
\hline
\end{tabular}

\section{Symmetric phase}

Another interesting phenomenon we have encountered during our study is the appearance of a symmetric phase just below $\beta_{\mathrm{c}}$ for all $N \geq 5$. That such phase exists in the vector $Z(6)$ spin model has been known for a long time [12], [3], [4]]. Here we confirm its existence for all vector $Z(N \geq 5)$ 
LGTs. The phase exhibits itself, e.g., in the distribution of magnetization angle. In the region just below critical point the magnetization angle is evenly distributed, showing that the continuous symmetry is not broken. Only at much smaller couplings one can observe the appearance of a symmetry-broken phase. In addition, we have studied the behavior of the population susceptibility below $\beta_{\mathrm{c}}$. For $N \geq 5$ it has a second broad maximum, which slowly moves to $\beta_{\mathrm{c}}$ with increasing lattice size. While, for $N=5$ the peak of the population susceptibility moves rather fast and practically collapses with the peak at the critical coupling on the largest available lattice $L=96$, for larger $N$ the peak stays rather far from the corresponding critical coupling, even for $L=96$ (with our data we cannot even exclude a situation when the convergence of the second maximum is logarithmic). We can imagine two scenarios to explain such behavior:

1. This symmetric phase exists only in finite volume. When the lattice size increases, the second maximum approaches the critical coupling and, eventually, the symmetric region shrinks and disappears. The explanation proposed in [13], ㄴ] might work in this case, too. Namely, the symmetric phase on the finite lattice is a phase with a very small mass gap and describes a crossover region to the symmetry-broken phase.

2. For $N>5$ the second maximum of the population susceptibility stays away from the critical couplings even in the infinite volume limit. In this case it might correspond to some higherorder phase transition and the symmetric phase with tiny or even vanishing mass gap exists also in the thermodynamic limit.

In both cases it is tempting to speculate that this symmetric region is reminiscent of the massless phase which appears in these models at finite temperature [17, [18]. Whichever scenario of the above two is realized, one needs to study the models on much larger lattices to uncover it.

\section{Summary}

We have studied the $3 D Z(N)$ LGT at zero temperature aiming at shedding light on the nature of phase transitions in these models for $N \geq 4$. This study was based on the exact duality transformations of the gauge models to generalized $3 D Z(N)$ spin models.

The main results can be shortly summarized as follows:

- We have determined numerically the position of the critical couplings for various $Z(N)$ models. For $N=2,3$ we find a reasonable agreement with the values quoted in the literature. For larger $N$, we have significantly improved the values given in [ए]]. Critical points obtained are in good correspondence with the results of our RG estimations [2]].

- The critical indices $v$ and $\eta$ derived here for $N=2,4$ suggest that these models are in the universality class of the $3 D$ Ising model, while our results for all $N>4$ hint all vector $Z(N \geq$ 5) LGTs belong to the universality class of the $3 D X Y$ model. The value of the index $v$ stays very close to the $X Y$ value, $v \approx 0.6716$, given in [ [20]. The index $\alpha$ in this case takes a small negative value. It thus follows that a third order phase transition takes place for $N \geq 5$.

- The index $\alpha$ extracted from the specific heat roughly agrees with the value of $3 D$ Ising model for all $N$ studied. The fact that we observe two different values of the index $\alpha$ dependently 
on whether we approach the critical point from below or from above leads to the conclusion that the first derivative of the free energy could exhibit a cusp in the thermodynamic limit if $N>4$.

- Our data also revealed the existence of a symmetric phase for all $Z(N)$ vector LGTs if $N>4$. However, substantially larger lattices are required to see if this phase survives the transition to the thermodynamic limit.

- The scaling $a T_{c}=\left(\beta_{\mathrm{c}}-\beta_{\mathrm{c}}\left(N_{T}\right)\right)^{-v} / N_{T}$ can be used to describe the dependence of finitetemperature critical points obtained in [[1]] on $N_{T}$.

\section{References}

[1] D. Horn, M. Weinstein and S. Yankielowicz, Phys. Rev. D 19 (1979) 3715.

[2] A. Ukawa, P. Windey, A.H. Guth, Phys. Rev. D 21 (1980) 1013.

[3] M.B. Einhorn, R. Savit, and E. Rabinovici, Nucl. Phys. B 170 (1980) 16.

[4] M. Caselle, M. Hasenbusch, M. Panero, JHEP 0301 (2003) 057; M. Caselle, M. Hasenbusch, Nucl. Phys. B 470 (1996) 435.

[5] R.V. Gavai, F. Karsch, B. Petersson, Nucl. Phys. B 322 (1989) 738.

[6] M. Fukugita, H. Mino, M. Okawa, A. Ukawa, J. Stat. Phys. 59 (1990) 1397.

[7] A. Bazavov, B.A. Berg, Phys. Rev. D 75 (2007) 094506.

[8] A. Bazavov, B.A. Berg, S. Dubey, Nucl. Phys. B 802 (2008) 421.

[9] M. Hellmund, W. Janke, Phys. Rev. E 74 (2006) 051113.

[10] G. Bhanot and M. Creutz, Phys. Rev. D 21 (1980) 2892.

[11] A. Polyakov, Nucl. Phys. B 120 (1977) 429; T. Banks, J. Kogut, R. Myerson, Nucl. Phys. B 129 (1977) 493; M. Göpfert, G. Mack, Commun. Math. Phys. 81 (1981) 97.

[12] P.D. Scholten, L.J. Irakliotis, Phys. Rev. B 48 (1993) 1291.

[13] S. Miyashita, J. Phys. Soc. Japan, 66 (1997) 3411.

[14] M. Oshikawa, Phys. Rev. B 61 (2000) 3430.

[15] O. Borisenko, V. Chelnokov, G. Cortese, R. Fiore, M. Gravina, A. Papa, I. Surzhikov, Phys. Rev. E 86 (2012) 051131.

[16] O. Borisenko, V. Chelnokov, G. Cortese, R. Fiore, M. Gravina, A. Papa, I. Surzhikov, PoS LATTICE 2012270 [arXiv:1212.1051 [hep-lat]].

[17] O. Borisenko, V. Chelnokov, G. Cortese, M. Gravina, A. Papa, I. Surzhikov, Nucl. Phys. B 870 (2013) 159.

[18] O. Borisenko, V. Chelnokov, G. Cortese, M. Gravina, A. Papa, I. Surzhikov, PoS LATTICE 2013463 [arXiv:1310.1039[hep-lat]].

[19] O. Borisenko, V. Chelnokov, G. Cortese, R. Fiore, M. Gravina, A. Papa, I. Surzhikov [arXiv:1310.5997[hep-lat]].

[20] M. Campostrini, M. Hasenbusch, A. Pelissetto, P. Rossi, E. Vicari, Phys. Rev. B 63 (2001) 214503.

[21] O. Borisenko, V. Chelnokov, V. Kushnir, PoS LATTICE 2013327. 